the critical amplitude of the pulsation and some dependences between physical parameters.

\title{
Astronomy and Astronomical Education in the FSU
}

Nikolai G. Bochkarev, Sternberg Astronomical Institute/Euroasian Astronomical Society, Universitetskij Pr. 13, 119899 Moscow, Russia

The current situation in astronomy and astronomical education over the territory of the Former Soviet Union is traced. New facilities for radio-astronomy are being put into work - the most important of them being the two coupled 32-m dishes, VLBI network "Quasar"; a number of observatories are acquiring an international status (in the frame of CIS); The Internet is becoming available for an increasing number of astronomical institutions. Azerbaijan astronomers have overcome their isolation from the rest of the world and cooperate actively with the astronomical community.

All-Russia and international Olympics in astronomy for high school students are held and attract participants from increasing number of regions of Russia and other states.

The outcome of the 9th JENAM in Moscow and of the events attached to the Meeting is presented.

\section{Conceptual Aproach to Astronomy and Basic Science Education}

M. Melek, Cairo University, Cairo, Egypt

An approach is developed, in which the major dynamical and physical concepts of astronomy and basic space science are used, to build a scheme (prototype model) for education on the undergraduate level. A way to teach different theories and observational facts is shown, in which those concepts are built in or used; within the suggested educational scheme. The computational techniques which are needed in astronomy and basic space science are disscussed at which steps through the suggested educational scheme might be introduced.

\section{Research Oriented Astrophysics Course for Physics Students}

Tapan K. Chatterjee and A Pedroza-Mendelez, Universidad A.Puebla A.P. 1316, Puebla PUE 72000, México 